\title{
GIFTS SM EDU Level 1B Algorithms
}

\author{
Jialin Tian ${ }^{a}$, Michael J. Gazarik ${ }^{b}$, Robert A. Reisse ${ }^{b}$, and David G. Johnson ${ }^{b}$ \\ ${ }^{a}$ SSAI, MS 468, NASA Langley Research Center, Hampton, VA 23681-2199; \\ ${ }^{b}$ NASA Langley Research Center, Hampton, VA 23681-2199
}

\begin{abstract}
The Geosynchronous Imaging Fourier Transform Spectrometer (GIFTS) Sensor Module (SM) Engineering Demonstration Unit (EDU) is a high resolution spectral imager designed to measure infrared (IR) radiances using a Fourier transform spectrometer (FTS). The GIFTS instrument employs three focal plane arrays (FPAs), which gather measurements across the long-wave IR (LWIR), short/mid-wave IR (SMWIR), and visible spectral bands. The raw interferogram measurements are radiometrically and spectrally calibrated to produce radiance spectra, which are further processed to obtain atmospheric profiles via retrieval algorithms. This paper describes the GIFTS SM EDU Level 1B algorithms involved in the calibration. The GIFTS Level 1B calibration procedures can be subdivided into four blocks. In the first block, the measured raw interferograms are first corrected for the detector nonlinearity distortion, followed by the complex filtering and decimation procedure. In the second block, a phase correction algorithm is applied to the filtered and decimated complex interferograms. The resulting imaginary part of the spectrum contains only the noise component of the uncorrected spectrum. Additional random noise reduction can be accomplished by applying a spectral smoothing routine to the phase-corrected spectrum. The phase correction and spectral smoothing operations are performed on a set of interferogram scans for both ambient and hot blackbody references. To continue with the calibration, we compute the spectral responsivity based on the previous results, from which, the calibrated ambient blackbody (ABB), hot blackbody (HBB), and scene spectra can be obtained. We now can estimate the noise equivalent spectral radiance (NESR) from the calibrated ABB and HBB spectra. The correction schemes that compensate for the fore-optics offsets and off-axis effects are also implemented. In the third block, we developed an efficient method of generating pixel performance assessments. In addition, a random pixel selection scheme is designed based on the pixel performance evaluation. Finally, in the fourth block, the single pixel algorithms are applied to the entire FPA.
\end{abstract}

Keywords: Remote sensing; Fourier transform spectrometry; Geosynchronous Imaging Fourier Transform Spectrometer (GIFTS); interferogram processing; radiometric calibration; Level 1B algorithms.

\section{INTRODUCTION}

The Geosynchronous Imaging Fourier Transform Spectrometer (GIFTS) Sensor Module (SM) Engineering Demonstration Unit (EDU) is a high resolution spectral imager designed to measure infrared (IR) radiances using a Fourier transform spectrometer (FTS). The GIFTS instrument employs three focal plane arrays (FPAs), which gather measurements across the long-wave $\left(685-1130 \mathrm{~cm}^{-1}\right)$ IR (LWIR), short/mid-wave $\left(1650-2250 \mathrm{~cm}^{-1}\right) \mathrm{IR}$ (SMWIR), and visible spectral bands. The raw interferogram measurements are radiometrically and spectrally calibrated to produce radiance spectra, which are further processed to obtain atmospheric profiles via retrieval algorithms. In this paper, we emphasize on the algorithmic components of the calibration process, which are divided in multiple blocks. Fig. 1 illustrates the basic data processing blocks for the GIFTS SM EDU Level 1B algorithms. In the raw data processing block, raw interferograms are modified based on the detector nonlinearity characteristics. A complex finite impulse response (FIR) filtering and decimation procedure is then performed on the interferograms. The next block of procedures includes the steps for calibrating a single interferogram. Prior to the radiometric calibration, the filtered and decimated complex interferograms must be phase-corrected so that their imaginary spectra contain only noise. The phase correction is performed on all three types of measurements for a series of scans, i.e., the ambient blackbody (ABB) reference, hot blackbody (HBB) reference, and scene scans. To reduce the random noise embedded in the reference measurements, a spectral smoothing

Further author information: (Send correspondence to Jialin Tian) Jialin Tian: E-mail: Jialin.Tian-1@nasa.gov, Telephone: 757-864-3698 
method can be applied to the phase-corrected blackbody reference spectra. Next, the calibrated ABB, HBB, and scene radiance spectra are computed based on the previous results. An assessment of the noise equivalent spectral radiance (NESR) is derived from the calibrated ABB and HBB references. Two additional correction algorithms are implemented to compensate for the fore-optics offsets and FPA off-axis effects. In the multi-pixel algorithm block, we introduce an efficient method of estimating the noise performance of the entire FPA, as well as a random pixel selection strategy that can be employed for generating a representative pixel sample set. In the FPA calibration block, the calibration algorithms introduced previously in the second block are performed on the entire FPA. The interferogram data at various stages are retrieved, linked, and stored via the storage and management system. Detailed descriptions for each data processing block are given in the following sections.

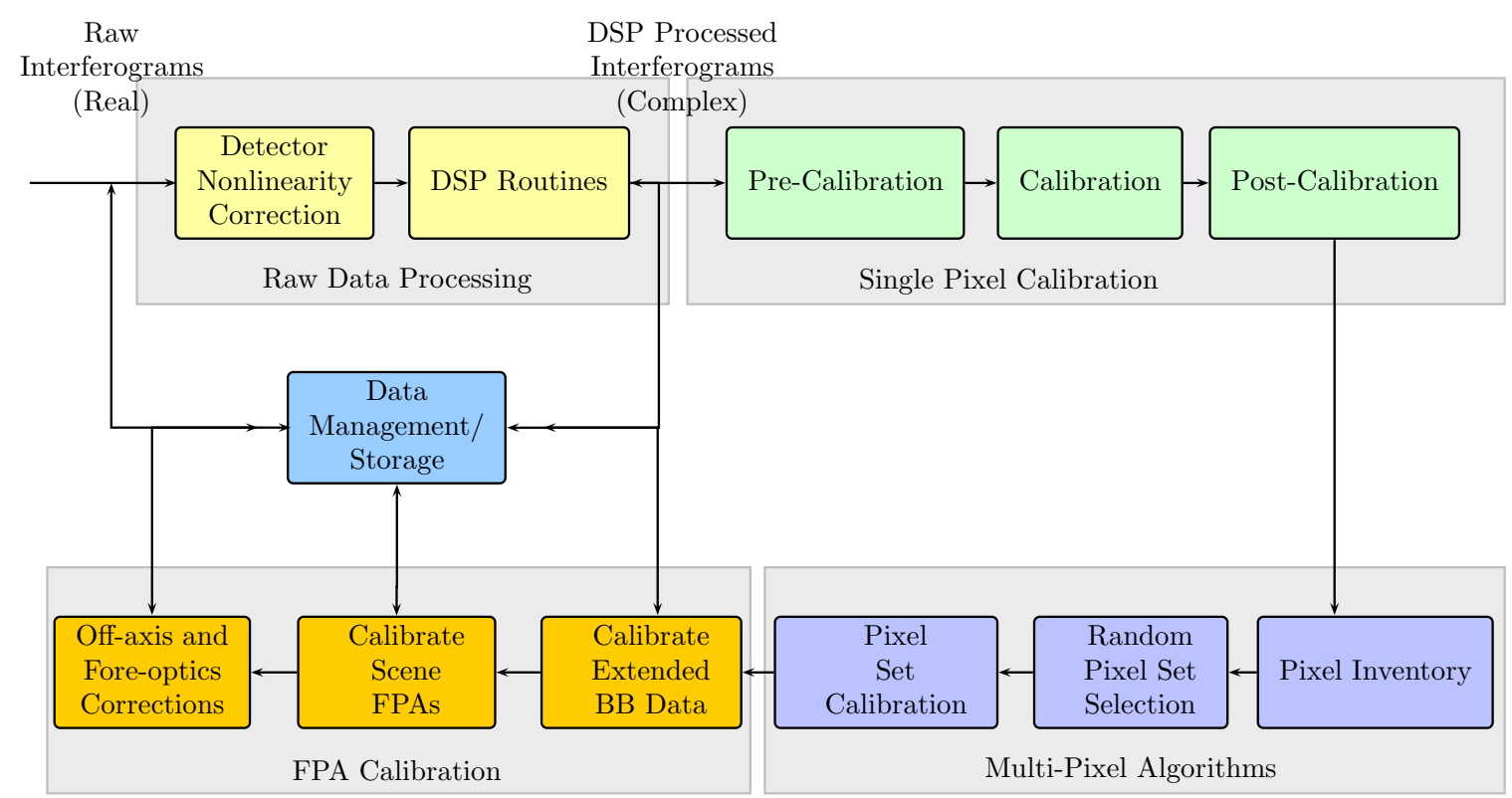

Figure 1. The basic GIFTS SM EDU data processing blocks.

\section{RAW DATA PROCESSING}

In this section, we introduce the necessary procedures to be performed on the measured raw interferograms prior to the calibration. We will discuss how the detector nonlinearity affects the raw interferogram data as well as the method used to characterize and correct the distortion. We then continue onto the topic of complexing filtering and decimation techniques, which have been applied to the interferogram data.

\subsection{Detector Nonlinearity Correction}

Ideally, a given input radiance function $I_{o}(n)$ and its corresponding measured output interferogram $I(n)$ are linearly proportional. However, in practice, this relationship becomes less ideal due to the distortion caused by the detector nonlinearity effect. The input-output function can be modeled as a $P^{t h}$-order polynomial

$$
y \approx F(x)=\sum_{p=0}^{P} a_{p} x^{p} .
$$

The polynomial function in Eq. (1) and its coefficients can be obtained from a set of known data points on the input-output curve, i.e., $\left(x_{1}, y_{1}\right),\left(x_{2}, y_{2}\right), \ldots,\left(x_{N-1}, y_{N-1}\right),\left(x_{N}, y_{N}\right)$. Fig. 2 depicts the effect of detector nonlinearity on the input $I_{o}(n)$. A sample polynomial curve with corresponding data pairs are also shown in the graph. The true amplitude of the input $I_{o}(n)$ can be estimated from the measured output $I(n)$ by inverting the polynomial function or by interpolation. To characterize the GIFTS detector nonlinearity, a series of small 




Figure 2. An illustration of the input-output distortion caused by the detector nonlinearity effect.

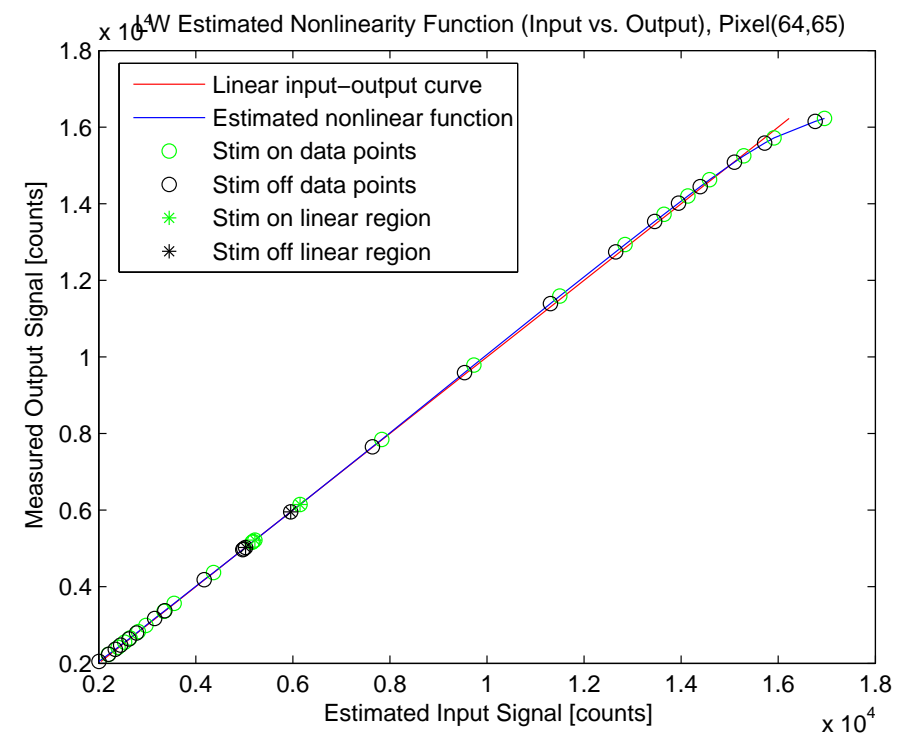

Figure 3. The detector nonlinearity characterization.

signal linearity measurements were made at several blackbody temperatures. At each given temperature, the output response data is collected. After injecting a stim source AC signal into the blackbody signal, the output response is re-measured. This pair of measurements gives us the quantity of $\Delta y=y_{o n}-y_{o f f}$. The variations in $\Delta y$ indicate nonlinear behaviors in the detector response function. However, we do not have any information on the corresponding input values other than the assumption that the stim source is constant. Consequently, an extra procedure is necessary before we can make the nonlinearity correction. The input levels must be estimated in order to construct the input-output curve. Fig. 3 shows the stim on and off data pairs with the ideal linear and estimated nonlinear input-output curves. The algorithm for estimating the nonlinearity curve is summarized in the following steps:

- Search for the linear region:

- Compute output small and large signals for all pairs of measurements. 
- Compute the differences between adjacent elements from the previous results.

- Compute the ratio $r_{n}$.

- The smallest value, $r_{k}^{*}$, in $r_{n}$ indicates that the smallest change in $\Delta y$, which implies that the most linear portion of the curve is located between $n=k$ and $n=k+1$.

- Estimate $\Delta x$.

- Linear region estimation:

- Estimate $x_{o f f, n}$ and $x_{o n, n}$ for $n=k, k+1$.

- Forward region estimation:

- For every pair of data above the linear region, we estimate their values from the previous pair using the finite difference approximation approach.

- Only one intercept point is constructed (one local extremum) to ensure the smoothness of the curve.

- The intercept point is placed at the midpoint between two adjacent pairs of data.

- Backward region estimation:

- Reverse the procedure for data pairs below the linear region.

Fig. 4 depicts three regions of the estimated curve. Once the detector nonlinear response function is constructed, the true input interferogram data can be found using interpolations.

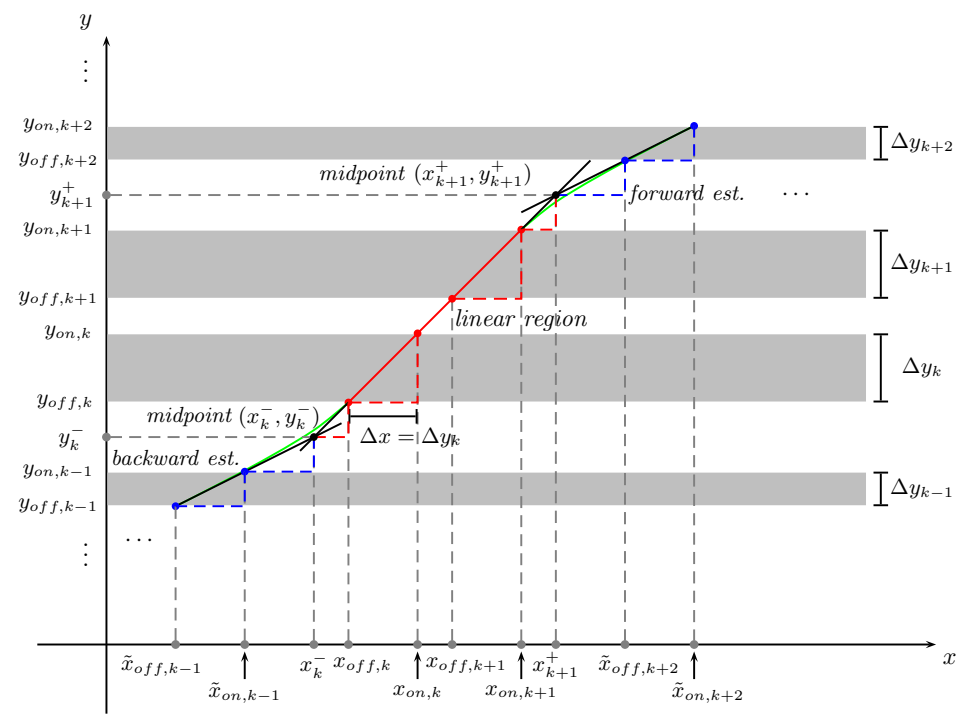

Figure 4. The estimation of the detector nonlinearity function.

\subsection{Decimation Filtering}

The next step involved in the raw data processing block is the procedure of complex filtering and decimation. For the GIFTS SM EDU data, we designed a linear-phase even-length complex finite impulse response (FIR) filter that has the form of

$$
h(n)=h_{r}(n)+j h_{i}(n), n=0, \ldots, N-1 .
$$

In the preceding expression, $h_{r}(n)$ contains the real part of the filter coefficients, which is the impulse response of an optimum equiripple linear-phase FIR filter. ${ }^{1}$ The imaginary part $h_{i}(n)$ is obtained by taking the Hilbert transform of $h_{r}(n)$. The output of the filter is complex in the interferogram domain, and does not contain an image spectrum in the frequency domain. The filter design parameters include: $\delta_{p^{-}}$passband ripple, $\delta_{s^{-}}$ 
stopband attenuation, $\omega_{p^{-}}$passband cutoff frequency, $\omega_{s^{-}}$stopband cutoff frequency, and $N$ - filter length. The Fourier transform of Eq. (2) can be written as

$$
H\left(e^{j \omega}\right)=H_{r}\left(e^{j \omega}\right)+j H_{i}\left(e^{j \omega}\right)
$$

where

$$
H_{r}\left(e^{j \omega}\right)=\mathcal{F}\left\{h_{r}(n)\right\} \text { and } H_{i}\left(e^{j \omega}\right)=\mathcal{F}\left\{h_{i}(n)\right\} .
$$

Next, we derive the imaginary impulse response $h_{i}(n)$ by taking the Hilbert transform of $h_{r}(n)$, for instance,

$$
h_{i}(n)=\mathcal{H}\left\{h_{r}(n)\right\}=h_{r}(n) * h_{h}(n) .
$$

The real and imaginary spectra can be expressed as

$$
\begin{aligned}
H_{r}\left(e^{j \omega}\right) & =\frac{1}{2}\left[H\left(e^{j \omega}\right)+H^{*}\left(e^{-j \omega}\right)\right] \text { and } \\
j H_{i}\left(e^{j \omega}\right) & =\frac{1}{2}\left[H\left(e^{j \omega}\right)-H^{*}\left(e^{-j \omega}\right)\right] .
\end{aligned}
$$

From the preceding expression, the imaginary spectrum also can be written as

$$
H_{i}\left(e^{j \omega}\right)= \begin{cases}-j H_{r}\left(e^{j \omega}\right), & 0<\omega<\pi \\ j H_{r}\left(e^{j \omega}\right), & -\pi \leq \omega<0\end{cases}
$$

or

$$
H_{i}\left(e^{j \omega}\right)=H_{h}\left(e^{j \omega}\right) H_{r}\left(e^{h \omega}\right)
$$

where

$$
H_{h}\left(e^{j \omega}\right)= \begin{cases}-j, & 0<\omega<\pi \\ j, & -\pi<\omega<0 .\end{cases}
$$

Note that $H_{h}\left(e^{j \omega}\right)$ is the spectrum of a Hilbert transformer, which can be seen as a 90-degree phase shifter.

\section{SINGLE-PIXEL CALIBRATION}

For a single pixel element on the FPA, the calibration procedures involve the following steps: phase correction, spectral smoothing, responsivity calculation, radiometric calibration, NESR assessments, fore-optics offsets adjustment, and off-axis effects correction.

\subsection{Phase Correction}

The phase correction algorithm is applied to the decimated and filtered complex interferogram $I_{m}(n)$. Its complex spectrum $N_{m}(\sigma)$ has the form of

$$
N_{m}(\sigma)=N(\sigma) e^{j \phi(\sigma)}+n(\sigma),
$$

where $n(\sigma)$ represents the noise vector, and $N(\sigma)$ is the real and noise-free spectrum. To estimate the phase function $\phi(\sigma)$, we adopt the Forman-Vanesse-Steel (FVS) method. ${ }^{2}$ In this technique, a Hamming window $w(n)$, of length $W$, is applied to the complex interferogram with respect to the zero path difference (ZPD), i.e., $n=n_{z p d}$. The windowed interferogram is written as $I_{w}(n)=w(n) I_{m}(n)$, where

$$
w(n)= \begin{cases}0.54-0.46 \cos \left(\frac{2 \pi n}{W-1}\right), & \left|n-n_{z p d}\right| \leq \frac{W-1}{2}, \\ 0, & \text { otherwise. }\end{cases}
$$

The resulting interferogram $I_{w}(n)$ is circular shifted to obtain $I_{w}^{\prime}(n)$ such that $I_{w}^{\prime}(0)=I_{w}\left(n_{z p d}\right)$. The spectral response of $I_{w}^{\prime}(n)$ is computed via the Fourier transform, i.e., $N_{w}^{\prime}(\sigma)=\mathcal{F}\left\{I_{w}^{\prime}(n)\right\}$, from which, the phase function can be estimated as $\hat{\phi}(\sigma)=\tan ^{-1} \frac{\operatorname{Im}\left(N_{w}^{\prime}(\sigma)\right)}{\operatorname{Re}\left(N_{w}^{\prime}(\sigma)\right)}$. By applying the phase estimate, the phase-corrected spectrum becomes

$$
\widehat{N}(\sigma)=N(\sigma) e^{j(\phi(\sigma)-\hat{\phi}(\sigma))}+n(\sigma) e^{-j \hat{\phi}(\sigma)},
$$

and its resulting real and imaginary components are given as

$$
\begin{aligned}
& \operatorname{Re}\{\hat{N}(\sigma)\} \approx N(\sigma)+\operatorname{Re}\{n(\sigma)\} \cos (\hat{\phi}(\sigma)), \\
& \operatorname{Im}\{\widehat{N}(\sigma)\} \approx \operatorname{Im}\{n(\sigma)\} \sin (\hat{\phi}(\sigma)) .
\end{aligned}
$$

Note that the imaginary part of the spectrum contains only noise, which can be discarded. 


\subsection{Spectral Smoothing}

The phase correction is performed on three types of spectra, i.e., ABB, HBB, and scene. An optional smoothing algorithm can be applied to the blackbody references to improve the noise performance of the calibrated radiances. Two smoothing techniques are being considered for this purpose: the least-squares smoothing filter and the moving average (MA) filter. The MA smoothing method approximates the function within the predefined window as a constant whereas the least-squares algorithm fits a polynomial to all points within the window. The MA method does not preserve higher order moments, however, it is less demanding computationally. See ${ }^{1,3}$ for detailed reviews of these two techniques. When the final noise performance of these two methods are compared, no significant differences were observed; therefore, the MA smoothing technique is chosen due to its simplicity. Fig. 5 (a) illustrates the phase corrected and smoothed longwave spectra for the real and imaginary blackbody references at ambient and hot temperatures, and Fig. 5 (b) shows the phase response functions after the correction.

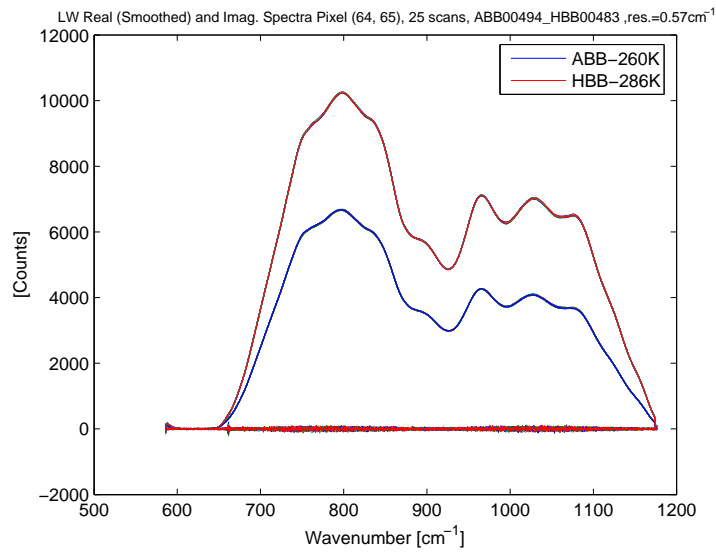

(a)

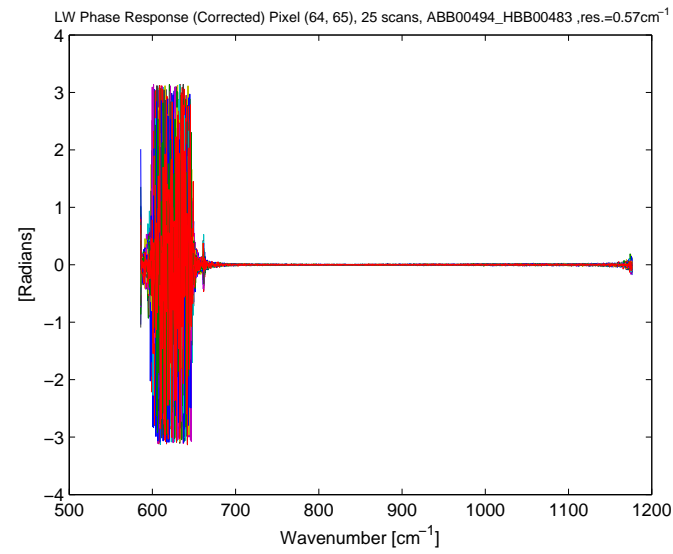

(b)

Figure 5. Phase corrected longwave blackbody spectra: (a) real and imaginary blackbody spectra at ambient (260K) and hot $(286 \mathrm{~K})$ temperatures, and (b) phase response functions.

\subsection{Radiometric Calibration}

To obtain the calibrated radiances, the first step involves the calculation of the spectral responsivity, which can be computed from

$$
R(\sigma)=\frac{\bar{N}^{H}(\sigma)-\bar{N}^{A}(\sigma)}{B^{H}(\sigma)-B^{A}(\sigma)} .
$$

$\bar{N}^{H}(\sigma)=\frac{1}{S} \sum_{i=1}^{S} N_{i}^{H}(\sigma)$ and $\bar{N}^{A}(\sigma)=\frac{1}{S} \sum_{i=1}^{S} N_{i}^{A}(\sigma)$ represent the mean HBB and ABB spectra averaged over $S$ scans, respectively. Using the relation of

$$
\bar{N}^{H}(\sigma)=R(\sigma)\left(B^{H}(\sigma)+O(\sigma)\right),
$$

the offset term can be solved from

$$
O(\sigma)=\frac{\bar{N}^{A}(\sigma) B^{H}(\sigma)-\bar{N}^{H}(\sigma) B^{A}(\sigma)}{\bar{N}^{H}(\sigma)-\bar{N}^{A}(\sigma)} .
$$

Next, the calibrated HBB, ABB, and scene radiance spectra can be determined, based on the responsivity and offset results, from

$$
\begin{aligned}
\widehat{B}_{i}^{H, A}(\sigma) & =\frac{N_{i}^{H, A}(\sigma)}{R(\sigma)}-O(\sigma), \\
\widehat{N}_{i}^{C}(\sigma) & =\frac{N_{i}^{C}(\sigma)}{R(\sigma)}-O(\sigma) .
\end{aligned}
$$


respectively. The variable $i$ denotes the scan number, i.e., $i=1,2, \ldots, S$.

\subsection{Noise Equivalent Spectral Radiance (NESR)}

This section describes the estimation of the noise equivalent spectral radiance (NESR) from calibrated blackbody references. The NESR is generally considered as a measure of the instrument noise performance, and can be estimated as the standard deviation of calibrated blackbody radiance spectra from multiple scans. The NESR definition is given by

$$
\mathrm{NESR}^{H, A}(\sigma)=\sqrt{\frac{1}{S} \sum_{i=1}^{S}\left(\widehat{B}_{i}^{H, A}(\sigma)-\widehat{B}^{H, A}(\sigma)\right)^{2}} .
$$

Fig. 6 depicts the smoothed HBB and ABB NESR estimates over their corresponding non-smoothed NESRs computed from 25 interferogram scans.



Figure 6. Smoothed and non-smoothed longwave HBB and ABB NESR estimates computed from 25 interferogram scans.

\subsection{Fore-optics Offsets}

The previous calibration results are computed using internal blackbody references. To compensate for the offsets generated from fore-optics, a correction scheme is incorporated into the previous model. This can be achieved by using additional data measurements collected from an extended source. Given $\bar{C}_{e}^{H}(\sigma)$ and $\bar{C}_{e}^{A}(\sigma)$ as calibrated extended blackbody sources averaged over all scans, we have

$$
\bar{C}_{e}^{H, A}(\sigma)=R_{e}(\sigma) B_{e}^{H, A}(\sigma)+O_{e}(\sigma) .
$$

By solving for $R_{e}(\sigma)$ and $O_{e}(\sigma)$, the actual scene radiances can be approximated as

$$
\widetilde{N}_{i}^{C}(\sigma)=\frac{\widehat{N}_{i}^{C}(\sigma)-O_{e}(\sigma)}{R_{e}(\sigma)} .
$$

Fig. 7 shows the calibrated longwave $\mathrm{HBB}, \mathrm{ABB}$, and scene radiance spectra after performing the fore-optics offsets correction. 


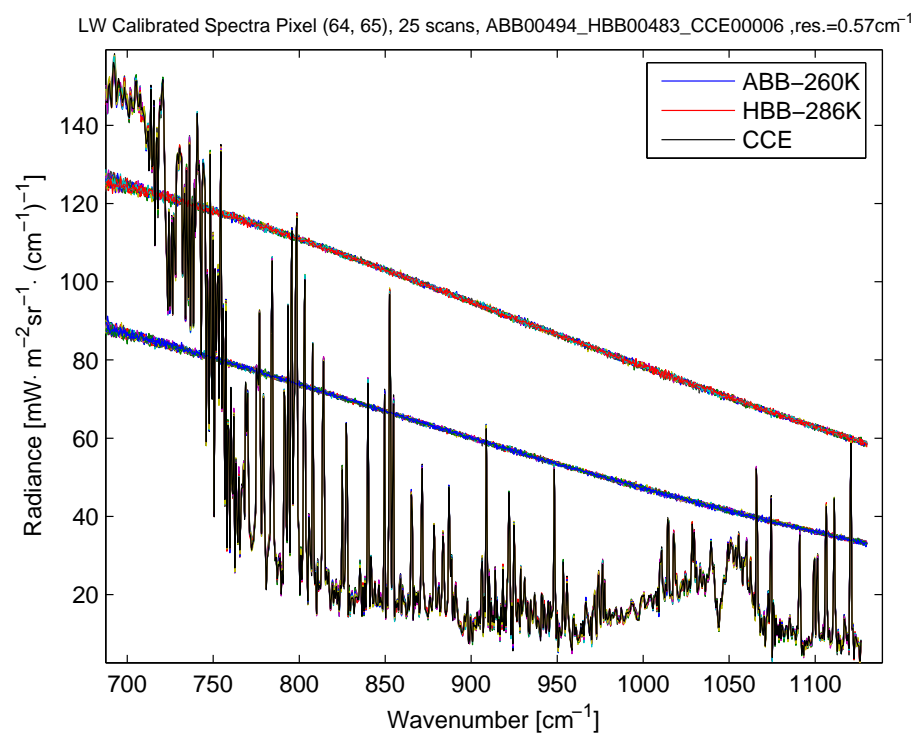

Figure 7. Calibrated longwave HBB, ABB, and scene radiance spectra after the fore-optics offsets correction (25 scans).

\subsection{Off-axis Effects}

In this section, the correction of the FPA off-axis effects is presented. Since the GIFTS FPA contains $128 \times 128$ detector elements, the distortion caused by the off-axis effects is too great to be neglected. The off-axis pixels are sampled at slightly shorter optical path differences (OPDs), which cause the spectra of these pixels to expand to slightly higher wavenumbers. ${ }^{4}$ The correction can be formulated as a fractional sampling rate conversion problem, which can be solved via sinc interpolations, i.e.,

$$
N_{i}^{C}(\sigma)=\sum_{m=0}^{M-1} \widetilde{N}_{i}^{C}\left(\sigma^{\prime}\right) \operatorname{sinc}\left(\sigma-\sigma^{\prime}\right), \quad \sigma^{\prime}=\frac{\sigma}{f},
$$

where $\sigma$ is the on-axis wavenumber scale, $\sigma^{\prime}$ is the off-axis scale, and $f$ is the off-axis factor that is computed from the FPA geometry. However, the sinc interpolation calculation is computational intensive, furthermore, the truncation window associated with the sinc kernels may cause overshoots in the resulting data. It has been shown that the zero-padding interpolation in the interferogram domain is equivalent to the sinc interpolation in the spectral domain except that the zero-padding method cannot evaluate the interpolation output at an arbitrary point. ${ }^{5}$ To overcome this limitation, we designed the method of "over-padding", in which, we assign an over-padding factor $g$ that is closely correlated to the final resolution of the corrected spectra. For instance, if $f=0.9977$, and the length of the spectrum is $N$, then the zero-padded interferogram length is the roundoff value of $g * N / f$. If $g=100$, the actual off-axis factor of 0.997702 is obtained; if $g=1$, then the actual value of $f$ becomes 0.99758 due to roundoff errors. The corrected spectrum can be obtained by downsampling the Fourier transform of the over-padded interferogram by the factor $g$. Fig. 8 (a) depicts the spectra for two pixels before the off-axis effects correction, one pixel is located near the center of the FPA whereas the other is situated near the corner of the FPA. A better wavenumber alignment was achieved after performing the off-axis effects correction as shown in Fig. 8 (b).

\section{MULTI-PIXEL ALGORITHMS}

We have discussed the algorithmic procedures required for a single pixel measurement. To evaluate the entire FPA without completing all of the calculation steps, we designed a pixel inventory method, which estimates the FPA responsivity and noise distributions. 


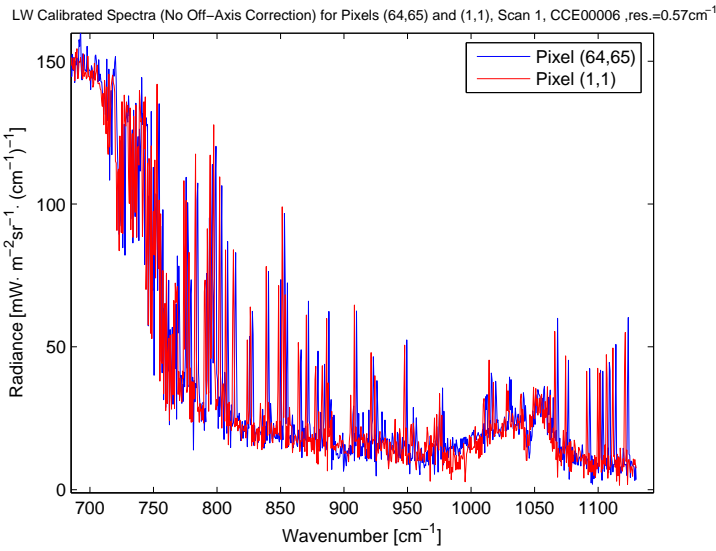

(a)



(b)

Figure 8. An illustration of the off-axis effects: (a) calibrated spectra for Pixel $(1,1)$ and Pixel $(64,65)$ before performing the off-axis effects correction, and (b) after the off-axis effects correction.

\subsection{Pixel Inventory}

The responsivity for any detector element can be approximated from the normalized intensity value at the ZPD, which is given by

$$
\widehat{R}^{x, y}=\frac{I_{m}^{x, y}\left(n_{z p d}\right)}{\frac{1}{X Y} \sum_{x} \sum_{y} I_{m}^{x, y}\left(n_{z p d}\right)} \forall x, y,
$$

where $x, y$ are pixel numbers. The FPA responsivity estimate is shown in Fig. 9 (a). The FPA responsivity distribution within a predefined range of $0.8 \leq \widehat{R}^{x, y} \leq 1.2$ is plotted in Fig. 9 (b). To assess the FPA noise, each

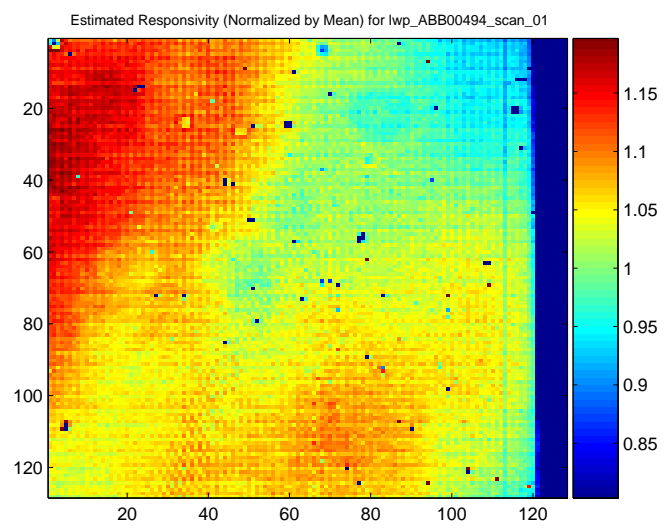

(a)

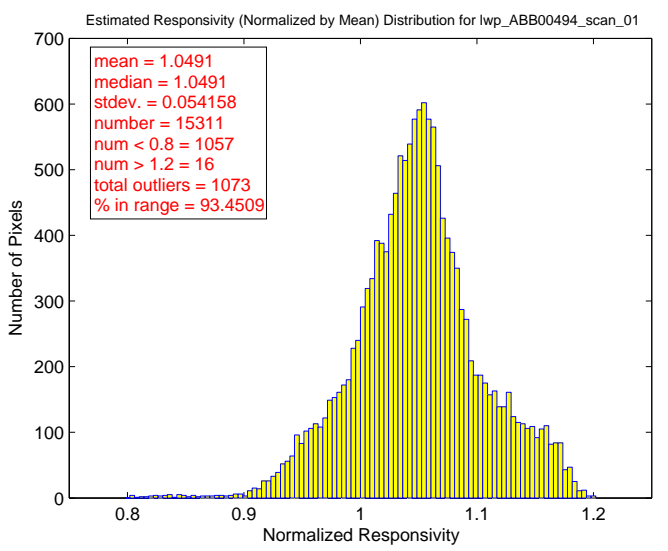

(b)

Figure 9. Longwave pixel inventory: (a) FPA responsivity estimates, and (b) FPA responsivity distribution plot. interferogram is first normalized by its value at $n=n_{z p d}$, i.e.,

$$
\widetilde{I}_{m}^{x, y}(n)=\frac{I_{m}^{x, y}(n)}{I_{m}^{x, y}\left(n_{z p d}\right)} \forall x, y
$$


Next, the estimated noise is computed from the root-mean-square value of the last $L$ samples in each interferogram that is represented by

$$
\widehat{N}^{x, y}=\sqrt{\frac{1}{L} \sum_{n=M-L}^{M-1}\left|\widetilde{I}_{m}^{x, y}(n)\right|^{2}} \forall x, y .
$$

Similarly, the FPA noise and its distribution are shown in Figs. 10 (a) and 10 (b), respectively.

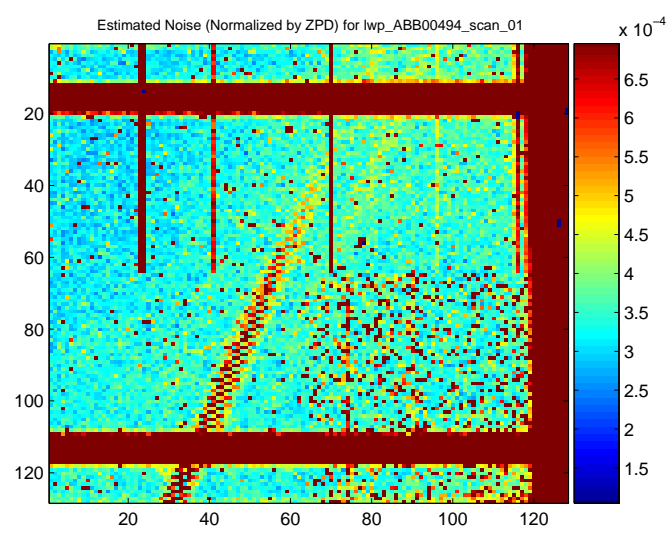

(a)



(b)

Figure 10. Longwave pixel inventory: (a) FPA noise estimates, and (b) FPA noise distribution plot.

\subsection{Random Pixel Set Generation}

Once we have the knowledge of the FPA responsivity and noise estimates, a set of selection criteria is established by defining an acceptable range for the responsivity and noise. Thus, a random sample set of pixels can be chosen such that it reflects the statistical properties of the entire FPA. We can then perform the actual calibration procedures on this sample set. Fig. 11 (a) shows a sample longwave pixel selection scheme, in which 64 random pixels are selected with 4 pixels per tap. The selection is uniformly distributed and unique within each tap. The NESR estimates of this sample pixel set can be seen in Fig. 11 (b).

\section{FOCAL PLANE ARRAY (FPA) CALIBRATION}

The calibration process for an entire FPA can be summarized into the following steps:

- Perform detector nonlinearity corrections for all pixels.

- Implement the complex filtering and decimation process for all pixels.

- Generate calibrated HBB and ABB data cubes from the extended blackbody source measurements.

- Calibrate the scene data cubes using the internal blackbody references and results obtained from the previous step.

- Compensate for the fore-optics offsets and off-axis effects.

Fig. 12 (a) shows a particular frame of calibrated radiances before applying the off-axis correction. The circular pattern illustrates the misalignment caused by the off-axis effects. Fig. 12 (b) demonstrates an improved spectral alignment after the correction. 


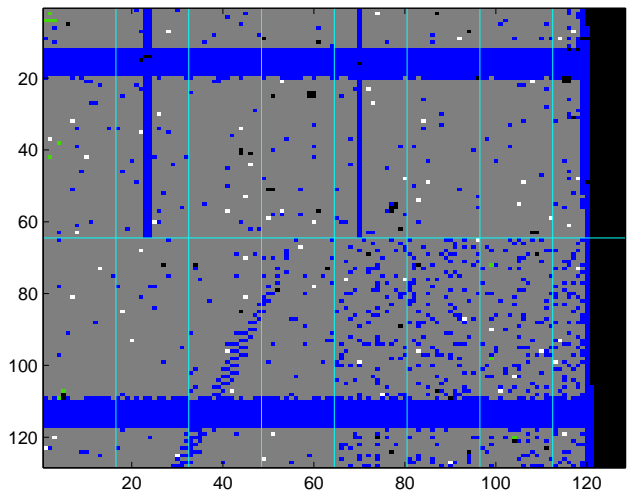

(a)

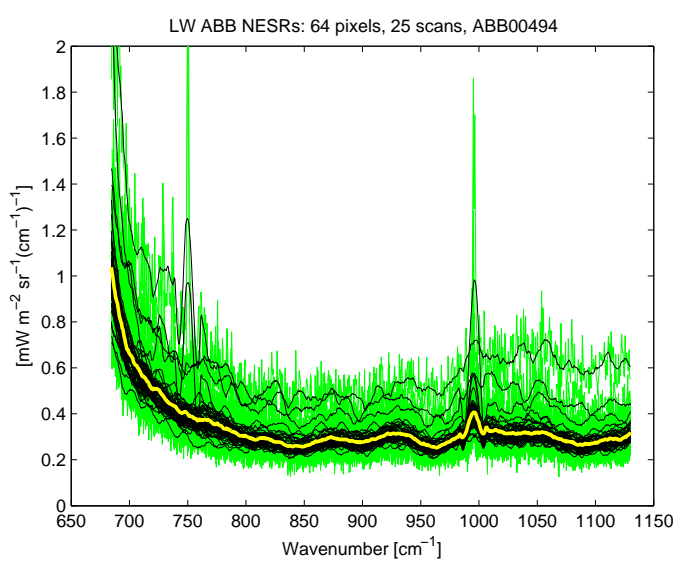

(b)

Figure 11. A sample longwave random pixel set: (a) blue pixels: out-of-range noise; green pixels: out-of-range responsivity; black pixels: out-of-range noise and responsivity; gray pixels: satisfy both noise and responsivity constraints; and white pixels: random pixel selection, and (b) NESR estimation results of the sample pixel set.

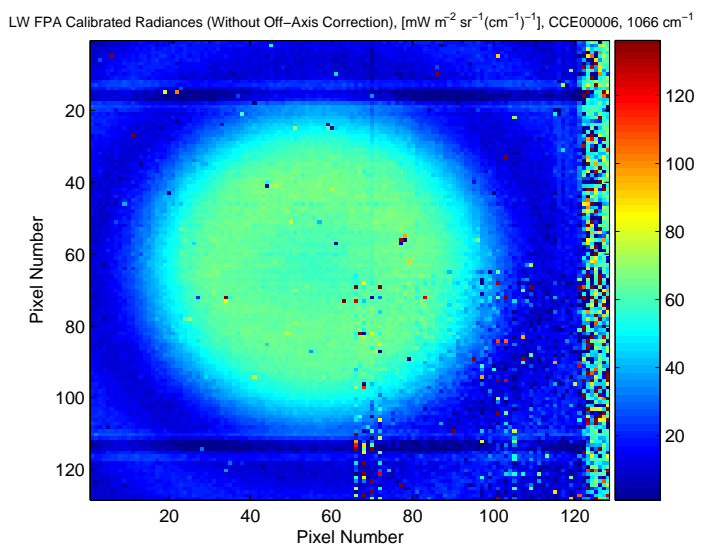

(a)

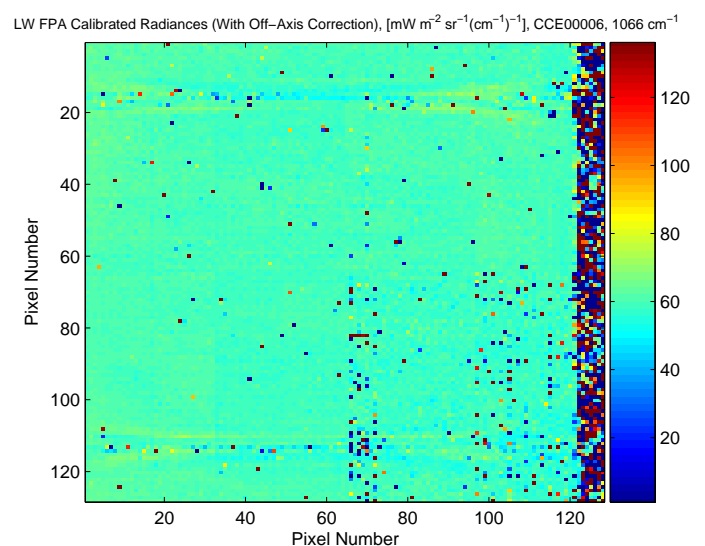

(b)

Figure 12. A calibrated longwave FPA frame: (a) before the off-axis effects correction, and (b) after the off-axis correction.

\section{CONCLUSION}

This paper describes Level 1B algorithms for calibrating the GIFTS SM EDU data spectrally and radiometrically. Four basic processing blocks were introduced. Initially, the raw interferograms must be corrected for the distortion caused by the detector nonlinearity, then the complex filtering and decimation procedure was performed. In the next block, the basic calibration steps were carried out. These include the procedures for phase correction, spectral smoothing, radiometric calibration, NESR computation, correction algorithms for the fore-optics offsets and the off-axis effects. The next two blocks of algorithms are concerned with multi-pixel processing techniques. Methods for estimating the FPA's performance, generating random pixel sample sets, and calibrating the complete FPA were discussed.

\section{REFERENCES}

1. A. V. Oppenheim, R. W. Schafer, and J. R. Buck, Discrete-Time Signal Processing, Prentice Hall, Upper Saddle River, 1999. 
2. M. L. Forman, W. H. Steel, and G. A. Vanasse, "Correction of asymmetric interferograms obtained in fourier transform spectroscopy," J. Opt. Soc. Amer. 56, pp. 59-63, 1966.

3. W. H. Press et al., Numerical Recipes in C: The Art of Scientific Computing, Cambridge University Press, Cambridge, 1992 (second edition).

4. J. Kauppinen and J. Partanen, Fourier Transforms in Spectroscopy, WILEY-VCH, Berlin, Germany, 2001.

5. P. J. La Rivière and X. Pan, "Mathematical equivalence of zero-padding interpolation and circular sampling theorem interpolation with implication for direct fourier image reconstruction," SPIE Conference on Image Processing 3338, pp. 1117-1126, 1998. 